\title{
Use of a murine endometriosis interna model for the characterization of compounds that effectively treat human endometriosis
}

\author{
CHRISTIANE OTTO $^{1}$, JENNY SCHKOLDOW ${ }^{1}$, ELISABETH KRAHL ${ }^{1}$, \\ IRIS FUCHS $^{1}$ and HANNES-FRIEDRICH ULBRICH ${ }^{2}$ \\ ${ }^{1}$ Therapeutic Research Group Oncology and Gynecological Therapy; \\ ${ }^{2}$ Global Drug Discovery Statistics, Bayer Pharma AG, Berlin 13342, Germany
}

Received October 6, 2011; Accepted November 29, 2011

DOI: $10.3892 / \mathrm{etm} .2011 .425$

\begin{abstract}
Endometriosis is a chronic, estrogen-dependent disease characterized by the presence of ectopic endometrium either in the pelvic cavity (endometriosis externa) or within the uterus (endometriosis interna, adenomyosis). Key symptoms are pelvic pain, dysmenorrhea and infertility. Established rodent animal models used for drug research in endometriosis have certain limitations. Since rodents do not menstruate, they cannot develop endometriosis externa spontaneously, but they suffer from endometriosis interna. There is growing evidence that human endometriosis externa and interna represent two faces of the same disease. Both are estrogen-dependent and respond to similar treatment paradigms. Here, we addressed the question whether a murine endometriosis interna model may also be suitable for the characterization of drugs employed in human endometriosis. We examined the effects of danazol, Faslodex and cetrorelix in SHN mice that developed endometriosis interna after pituitary grafting. The GnRH antagonist cetrorelix and the estrogen receptor antagonist Faslodex, which negatively interfered with estrogen-mediated signaling, completely inhibited endometriosis interna, whereas danazol, an androgenic progestin, showed significant therapeutic activity in the majority of SHN mice. We conclude that this murine endometriosis interna model may be a valuable complement to established endometriosis externa models to support drug research in human endometriosis.
\end{abstract}

\section{Introduction}

Five to fifteen percent of women in their reproductive age suffer from endometriosis, an estrogen-dependent, chronic

Correspondence to: Dr Christiane Otto, Therapeutic Research Group (TRG), Oncology and Gynecological Therapy, Bayer Pharma AG, Muellerstrasse 178, Berlin 13342, Germany

E-mail: christiane.otto@bayer.com

Key words: endometriosis externa, endometriosis interna, adenomyosis, mouse models disease characterized by the presence of ectopic endometrium in either the pelvic cavity or the uterus (1). Main symptoms are pelvic pain, dysmenorrhea, dyspareunia and infertility (2). According to the localization of endometriotic lesions, endometriosis can be divided into endometriosis interna (presence of ectopic endometrium within the myometrium) and endometriosis externa (presence of ectopic endometrium within the pelvic cavity) (3). Formerly, endometriosis interna and externa were perceived as one pathological entity characterized by mucosal invasions and termed adenomyoma (4). In 1927, the theory of retrograde menstruation was generated and served as a potential pathophysiological explanation for the development of endometriosis externa and, as a consequence, led to the separation of endometriosis externa and interna (5). Nowadays, there is again increasing evidence that endometriosis interna and externa may represent different phenotypes of the same disease $(3,4)$. The tissue-injury and repair theory suggests that local hyperestrogenism in response to microtraumatization at the endometrial-myometrial interface leads to enhanced uterine peristaltic activity within the endomyometrial junctional zone (6). As a consequence, dislocated basal endometrium either infiltrates the myometrium (adenomyosis, endometriosis interna) or reaches via the ovarian tubes the peritoneal cavity leading to endometriosis externa (6).

Since endometriosis significantly impairs quality of life of severely affected women, there is a continuous medical need for the development of new treatment paradigms. Currently, besides laparoscopy, progestins, oral contraceptives, GnRH analogues, danazol, as well as pain medication and other experimental approaches, such as COX-2 inhibitors, aromatase inhibitors, selective estrogen receptor modulators and GnRH antagonists, are employed $(3,7)$. Animal models that are used in early stages of drug testing often rely on nonmenstruating rodents with induced endometriosis-like lesions. In homologous models, normal endometrial tissue is surgically transplanted into the peritoneal cavity of immunocompetent recipients and starts to grow in an estrogen-dependent manner. In heterologous models, human endometriotic lesions are transplanted into the peritoneal cavity of immunodeficient mice $(8,9)$. In both models, drug candidates are analysed with regard to their ability to influence the estrogen-dependent 
growth of the endometrial or endometriotic transplants. Apart from rodents, primates that spontaneously develop endometriosis or that have been transplanted intraperitoneally with endometrium can be used to study drug candidates (8). However, primate studies are expensive and do not allow for high throughput analysis in early stages of drug discovery. On the other hand, the established endometriosis externa models in rodents are under discussion. It remains to be established whether the use of immunodeficient mice or whether the transplantation of normal endometrium into the peritoneal cavity of a non-menstruating species indeed completely reflects all pathophysiological aspects of human endometriosis. Additional models, that complement the established battery of endometriosis externa models, may therefore be helpful.

Taking into account that human adenomyosis and endometriosis are again perceived as two phenotypes of the same disease $(3,4)$, we used a previously described murine endometriosis interna model (10) and addressed the question of whether this model is suitable for the characterization of drug candidates that effectively treat human endometriosis. We analysed three different compounds in this model: danazol, an androgenic progestin that was widely used in the clinic in the 1980s (11), cetrorelix, a GnRH antagonist that was successfully employed in human experimental studies (11), and the antiestrogen Faslodex (ICI182780), which we used as a tool compound to examine the estrogen dependency of the disease.

\section{Materials and methods}

Chemicals. Danazol, Faslodex (ICI182780) and cetrorelix were synthesized in the Chemistry Department of Bayer Pharma AG (Berlin, Germany).

Animals. SHN mice were kindly provided by the Japanese RIKEN BRC Institute, and the SHN breeding colony was maintained at Taconic (Denmark). Mice were kept on a 14-h light/10-h dark cycle and provided with food and water ad libitum. All animal procedures were carried out according to German animal welfare law with the permission of the District Government of Berlin.

Endometriosis interna model using SHN mice. To increase the incidence of endometriosis interna in adult SHN mice, we grafted 1 male donor pituitary under the kidney capsule of 8-week-old female mice as described previously (10). Ten randomly chosen control mice remained unoperated. Two weeks after pituitary grafting, 40 operated animals were randomly allocated into four groups. They remained either untreated or were treated subcutaneously on 6 days per week with danazol ( $25 \mathrm{mg} / \mathrm{kg}$ body weight, dissolved in sesame oil), Faslodex $(5 \mathrm{mg} / \mathrm{kg}$ body weight, dissolved in ethanol/arachisoil, 10/90\%, v/v) or cetrorelix (100 $\mu \mathrm{g} /$ mouse, dissolved in water containing $5 \%$ mannitol). Treatment was performed for 8 weeks. The experiment encompassed the following groups $(\mathrm{n}=10)$ : no pituitary grafting, no treatment; pituitary grafting, no treatment; pituitary grafting, danazol treatment $(25 \mathrm{mg} / \mathrm{kg}$ body weight); pituitary grafting, Faslodex treatment $(5 \mathrm{mg} / \mathrm{kg}$ body weight); pituitary grafting, cetrorelix treatment $(100 \mu \mathrm{g}$ per mouse).
Animals were sacrificed on day 73 after pituitary grafting and relative uterine weights were determined. The uterine horns were fixed overnight in $4 \%$ formalin in phosphate buffered saline (PBS). Afterwards, the tissue was dehydrated and embedded in paraffin. Sections $(5-\mu \mathrm{m}$ thick) were prepared and deparaffinized. For antigen retrieval, sections were heated in citrate buffer (S2031; Dako) at $900 \mathrm{~W}$ and boiled for $10 \mathrm{~min}$ at $140 \mathrm{~W}$. Endogeneous peroxidase was blocked by incubation with $3 \%$ hydrogen peroxide in PBS at room temperature for $10 \mathrm{~min}$. For actin staining, a mouse monoclonal anti-human $\alpha$-smooth muscle actin antibody (GTX18147, dilution 1:150; Genetex) and a secondary horseradish-peroxidase-coupled anti-mouse antibody were employed according to the manufacturer's instructions (Genetex). For nuclear counterstain, sections were stained with hematoxylin, dehydrated and embedded with Eukitt. Disease severity was assessed by an investigator blinded to the experimental treatment the animals had received. Four transverse uterine sections per animal were analysed using a modification of a previously developed scoring system (12). The adenomyosis score is a six-orderedlevel scoring system that reflects the degree of myometrial infiltration by the endometrium: 0 , no signs of endometriosis interna; 1, the inner circular myometrial layer looses its concentricity; 2, endometrial stroma and glands invade the inner circular layer of the myometrium; 3, endometrial stroma and glands are located between the inner circular and outer longitudinal myometrial layer; 4, endometrial stroma and glands infiltrate the outer myometrial layer; 5, endometrial stroma and glands pass the outer myometrial layer and have direct contact with the peritoneum.

Statistical analysis. For the statistical evaluation of the disease score, the pituitary-grafted group was considered as reference against which all other experimental groups were compared at the 5\% significance level. Primary endpoint was the disease score as a measurement on an ordinal scale. Comparisons against the respective reference group were made using Dunn's procedure which controls for the familywise error rate (13).

Results for relative uterine weights are depicted as means \pm standard deviation. The experimental groups were compared to animals that did not receive a pituitary again using a significance level of 5\%. For data evaluation a log-normal distribution is a suitable model. Therefore, Dunnett's test (14) was applied on logarithmized data, keeping the familywise error rate under control. Since the performed experiments were exploratory in nature, no across variable $\alpha$-adjustments were applied.

\section{Results}

Adult SHN mice remained unoperated or received pituitary isografts under the kidney capsule. Two weeks after transplantation, animals remained either untreated or were treated for 8 weeks with danazol, Faslodex or cetrorelix. Representative images of uteri from the different treatment groups are shown at the same magnification (Fig. 1). The results of the individual animals in the different treatment groups were also evaluated using the scoring system described in Materials and methods (Fig. 2A). Briefly, a score of 0 was attributed to healthy animals, whereas higher score numbers correlated with the invasion depth of endometrial stroma and glands into 


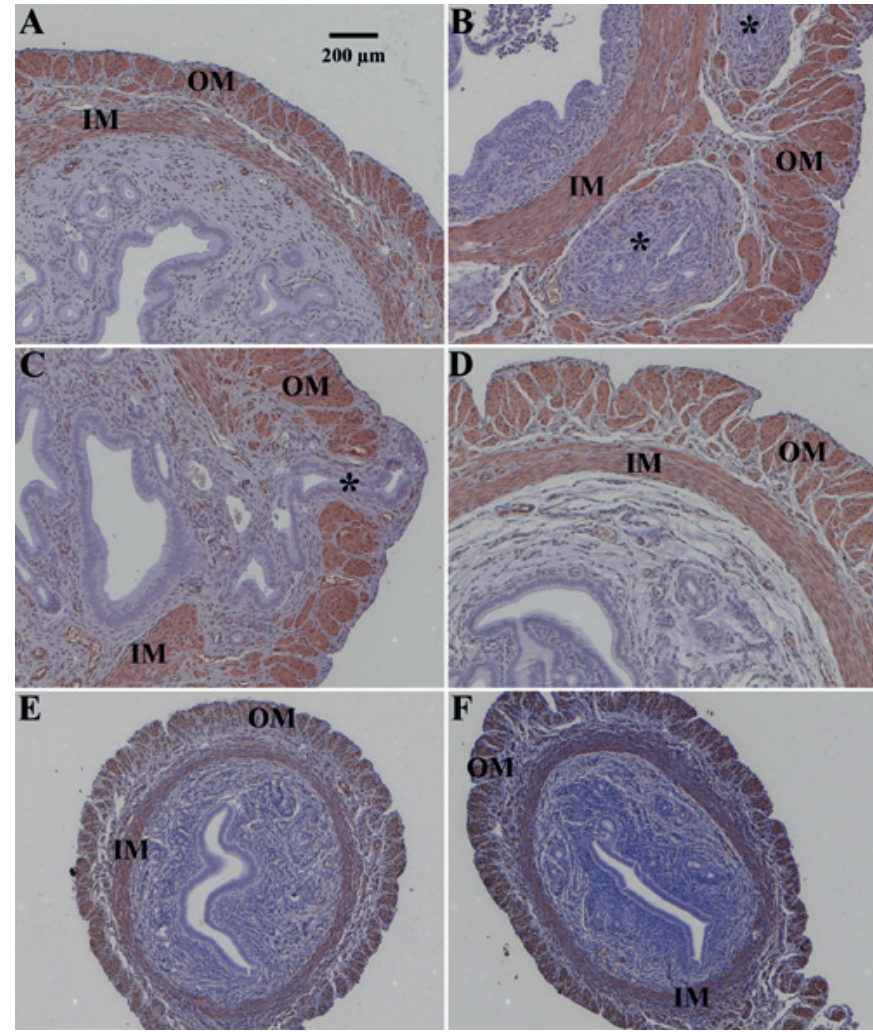

Figure 1. Uterine sections. Animals (A and B) remained unoperated or (C-F) received a pituitary graft under the kidney capsule. Two weeks later, animals (A-C) remained either untreated or were treated for 8 weeks with (D) danazol, (E) Faslodex or (F) cetrorelix. Asterisks indicate endometriotic foci invading the inner (IM) and/or outer muscular (OM) layer of the uterus. (A) Untreated control animals were either healthy or (B) suffered from severe endometriosis interna. (C) Pituitary grafting led to endometriosis interna in all animals. (D) Danazol treatment prevented endometriosis interna in the majority of animals. (E) Faslodex and (F) cetrorelix inhibited the disease in all animals. All images were taken at the same magnification.

the uterine smooth muscular layers. The median score of each treatment group is depicted in Fig. 2A as a horizontal bar. Unoperated control animals were either healthy (Figs. 1A and 2A) or suffered from severe endometriosis interna (Figs. 1B and 2A). Asterisks in Fig. 1 indicate endometrial glands and stroma invading the inner (IM) and/or outer muscular (OM) layer of the uterus. This heterogeneous disease activity in unoperated animals (Fig. 1A vs. B; Fig. 2A) was described previously.

Mice of the SHN strain tend to develop endometriosis interna spontaneously with increasing age (15), and pituitary grafting in these animals is known to facilitate homogeneous disease development at earlier time points. Pituitary grafting provoked endometriosis interna in all animals (Figs. 1C and 2A) leading to a median disease score of 4 (Fig. 2A). As an example, the most severe form of endometriosis interna reflecting a score of 5 is depicted (Fig. 1C). Endometrial glands and stroma passed through the outer muscular layer of the uterus (Fig. 1C). Sixty percent of the animals treated with danazol after pituitary isografting were healthy; the median disease score after danazol treatment was 0 ( $p<0.05$ vs. grafted animals) (Fig. 2A). An example of successful danazol treatment is depicted in Fig. 1D. Faslodex (median disease score $0, p<0.05$ vs. grafted animals; Fig. 1E) and cetrorelix

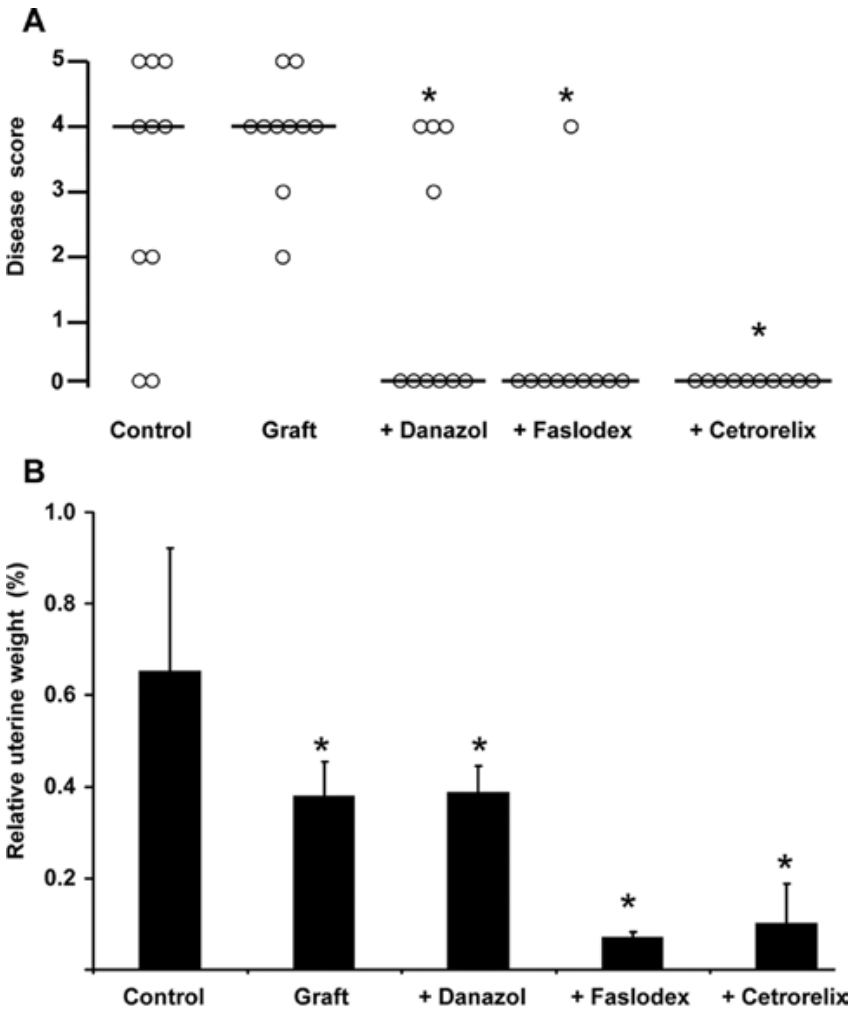

Figure 2. Disease scores and relative uterine weights. SHN mice remained unoperated (control) or received pituitary grafts followed by subsequent treatment with danazol, Faslodex or cetrorelix. Median disease scores are indicated as horizontal bars, a score of 0 indicates healthy animals. Asterisks indicate scores significantly different from the score of the pituitary-grafted group ( $\left.{ }^{*}<0.05\right)$. (A) Uteri from unoperated control animals exhibited disease heterogeneity. Pituitary grafting provoked endometriosis interna in all animals. Danazol (median score 0, p<0.05 vs. pituitary-grafted group) faslodex (median score $0, p<0.05$ vs. pituitary-grafted group) and cetrorelix (median score $0, p<0.05$ vs. pituitary-grafted group) prevented the disease in pituitary-grafted animals. (B) Compared to the untreated control animals, uteri of pituitary-grafted animals exhibited reduced relative weight due to increased progestogenic activity. Danazol application did not further reduce relative uterine weight. Faslodex and cetrorelix abolished estrogenic activity in the uterus and severely reduced relative uterine weight. Data are given as the means \pm standard deviation. ${ }^{*} \mathrm{p}<0.05$ (vs. the control group).

(median disease score $0, \mathrm{p}<0.05$ vs. grafted animals; Fig. 1F) successfully prevented endometriosis interna development after pituitary isografting in SHN mice. Only 1 animal treated with Faslodex still suffered from the disease (Fig. 2A). Relative uterine weights in the unoperated control group showed a high standard deviation, since the animals were in random cycle stages (Fig. 2B). Pituitary isografting resulted in significantly lower uterine weights (Fig. 2B). This finding was in line with previous reports demonstrating that pituitary isografting led to increased progesterone levels and smaller uteri in pituitarygrafted compared to unoperated animals (16). Progesterone inhibits the proliferative activity of estradiol in the endometrium and therefore reduces uterine weight. Consequently, additional treatment with the androgenic progestin danazol also resulted in lower uterine weights compared to unoperated control animals (Fig. 2B). The 'antiestrogenic' drugs Faslodex (estrogen receptor antagonist) and cetrorelix (GnRH receptor antagonist leading to inhibition of estradiol synthesis) strongly reduced relative uterine weight (Fig. 2B) and size (Fig. 1E and 
F vs. Fig. 1A-D) by completely abolishing estrogenic effects in the uterus. Faslodex-treated animals suffered from ovarian cysts (data not shown), a phenotype that was also observed in estrogen receptor $\alpha$-deficient mice (17). Since Faslodex completely inhibited estrogen receptor-mediated signaling and prevented the negative feedback regulation of estradiol within the hypothalamic-gonadal axis, ovaries were hyperstimulated and developed cysts.

In conclusion, our data provided evidence that Faslodex and cetrorelix are fully effective for the treatment of endometriosis interna in mice, whereas danazol showed efficacy in the majority of treated animals.

\section{Discussion}

The aim of the present study was to evaluate whether a murine endometriosis interna model could supplement established rodent endometriosis externa models for drug research in human endometriosis. For that purpose, we analysed three compounds that have been employed either clinically or in experimental studies in human endometriosis $(11,18)$. The GnRH antagonist cetrorelix and the estrogen receptor antagonist Faslodex, both interfering negatively with estradiol-mediated signaling, completely suppressed the disease. Danazol, an androgenic progestin, inhibited endometriosis interna in the majority of animals as reported previously (19).

To date, rodent endometriosis externa models are widely used in drug research, but may have limitations and may not mimick all aspects of human pathophysiology. For example, in homologous rodent models, 'healthy' uterus is cut into fragments and transplanted into the peritoneum $(8,9)$, whereas it has been suggested that the eutopic endometrium of women suffering from endometriosis may already be abnormal (20). Nude mice lacking an intact immune system are employed in the heterologous model which cannot mimick the inflammatory response normally seen in human endometriotic lesions (9). Although heterologous rodent endometriosis externa models, such as human endometriosis, are responsive towards drugs and manipulations that induce a hypoestrogenic state, such as ovariectomy, GnRH agonists, aromatase inhibitors, danazol and selective estrogen receptor modulators (21), there may be difficulties with the analysis of novel target families in which, for example, the murine ligand does not bind to the receptor of the human transplant. In some aspects, murine endometriosis interna models may have some advantages compared to rodent endometriosis externa models: i) the endometrium becomes invasive and, thus, does not appear healthy any longer; ii) the mice have an intact immune system; and iii) there is no problem with species selectivity of certain receptor ligands.

Identical to endometriosis externa, endometriosis interna is an estrogen-dependent disease and can be induced in several non-menstruating species, such as mice, rabbits and guinea pigs, after long-term estradiol treatment (22). Pituitary grafting is known to stimulate an increase in prolactin, growth hormone and progesterone (15). Ovariectomy of SHN mice, prevented adenomyosis establishment (23), indirectly demonstrating the estrogen-dependency of this model. In addition, the estrogendependency of the SHN model was further substantiated by our finding that Faslodex and cetrorelix suppressed endometriosis interna.
Progestins are marketed drugs for the treatment of endometriosis (24). However, as reported previously (19) danazol, an androgenic progestin, was only effective in the majority of affected SHN mice. In the established homologous endometriosis externa model, even weaker progestin responsivity was observed (25). Wild-type mice were ovariectomized and fragments of one uterine horn were transplanted into the peritoneum. There was a strong increase in lesion volume after estradiol treatment (from $25.8 \mathrm{~mm}^{3}$ in the untreated group to $59.3 \mathrm{~mm}^{3}$ in the estradiol-treated group) demonstrating estrogen dependency of the model. Compared to the estradiol effects, the therapeutic progesterone effects, although statistically significant, seemed to be minor; compared to untreated animals, progesterone decreased lesion volume from 25.9 to $23.7 \mathrm{~mm}^{3}$ and, compared to estradiol-only treatment, progesterone plus estradiol treatment diminished lesion volume from 66.8 to $59.3 \mathrm{~mm}^{3}$ (25). Notably, a significant portion of endometriosis patients exhibits progesterone resistance and does not respond to progestin treatment (26). The employed endometriosis interna model reflected the partial progestin responsivity seen in the human situation. Further research is required to understand the molecular mechanisms of treatment resistance towards progestins in endometriosis.

Taken together, although still under debate, the perception of endometriosis interna (adenomyosis) and externa as two phenotypes of the same disease is growing $(3,4)$. In humans, endometriosis interna and externa respond to the same medical treatment paradigms (27). This may reflect that similar pathophysiological processes stimulate the development of endometriosis interna and externa in women. The results of our study suggest that it may be worthwhile to exploit murine endometriosis interna models for drug research in human endometriosis. Endometriosis interna models could represent a valuable complement to the existing homologous and heterologous endometriosis externa models.

\section{Acknowledgements}

The authors are very grateful to the Japanese RIKEN BioResource Center which kindly provided the SHN mouse strain. They also wish to thank Tanja Lehmann and Lam Cam Quoc from Bayer Pharma AG, Berlin, Germany, for performing the pituitary grafting in the mice. They thank Professor Daniel Medina (Baylor College of Medicine, Houston, TX, USA) for the helpful discussions and technical instructions regarding the pituitary grafting.

\section{References}

1. Leyendecker G, Herbertz M, Kunz G and Mall G: Endometriosis results from the dislocation of basal endometrium. Hum Reprod 17: 2725-2736, 2002.

2. Kitawaki J, Kado N, Ishihara H, Koshiba H, Kitaoka Y and Honjo H: Endometriosis: the pathophysiology as an estrogendependent disease. J Steroid Biochem Mol Biol 83: 149-155, 2002.

3. Halis G, Mechsner S and Ebert AD: Diagnosis and treatment of deep infiltrating endometriosis. Dtsch Arztebl Int 107: 446-455, 2010.

4. Benagiano G, Brosens I and Carrara S: Adenomyosis: new knowledge is generating new treatment strategies. Womens Health 5: 297-311, 2009.

5. Sampson JA: Peritoneal endometriosis due to the menstrual dissemination of endometrial tissue into the peritoneal cavity. Am J Obstet Gynecol 14: 422-469, 1927. 
6. Leyendecker G, Wildt L and Mall G: The pathophysiology of endometriosis and adenomyosis: tissue injury and repair. Arch Gynecol Obstet 280: 529-538, 2009.

7. Fedele L, Somigliana E, Frontino G, Benaglia L and Vigano P: New drugs in development for the treatment of endometriosis. Expert Opin Investig Drugs 17: 1187-1202, 2008.

8. Story L and Kennedy S: Animal studies in endometriosis: a review. ILAR J 45: 132-138, 2004

9. Tirado-Gonzalez I, Barrientos G, Tariverdian N, Arck PC, Garcia MG, Klapp BF and Blois SM: Endometriosis research: animal models for the study of a complex disease. J Reprod Immunol 86: 141-147, 2010

10. Mori $\mathrm{T}$ and Nagasawa $\mathrm{H}$ : Mechanism of development of prolactin-induced adenomyosis in mice. Acta Anat 116: 46-54, 1983.

11. Rodgers AK and Falcone T: Treatment strategies for endometriosis. Expert Opin Pharmacother 9: 243-255, 2008.

12. Mori T, Kyokuwa M and Nagasawa H: Animal model of uterine adenomyosis: induction of the lesion in rats by ectopic pituitary isografting. Lab Anim Sci 48: 64-68, 1998.

13. Dunn OJ: Multiple comparisons using rank sums. Technometrics 6 : 241-252, 1964.

14. Dunnett CW: A multiple comparison procedure for comparing several treatments with a control. J Am Statist Assoc 50: 1096-1121, 1955.

15. Singtripop T, Mori T, Shiraishi K, Park MK and Kawashima S: Age-related changes in gonadotrophin, prolactin and growth hormone levels with reference to the development of uterine adenomyosis in female SHN mice. In Vivo 7: 147-150, 1993.

16. Huseby R, Soares MJ and Talamantes F: Ectopic pituitary grafts in mice: hormone levels, effects on fertility, and the development of adenomyosis uteri, prolactinomas, and mammary carcinomas. Endocrinology 116: 1440-1448, 1985.

17. Couse JF, Yates MM, Walker VR and Korach K: Characterization of the hypothalamic pituitary-gonadal axis in estrogen receptor (ER) null mice reveals hypergonadism and endocrine sex reversal in females lacking ER $\alpha$ but not ER $\beta$. Mol Endocrinol 17: $1039-1053,2003$
18. Guo SW and Olive DL: Two unsuccessful clinical trials on endometriosis and a few lessons learned. Gynecol Obstet Invest 64: 24-35, 2007.

19. Singtripop T, Mori T, Sakamoto S, Sassa S, Park MK and Kawashima S: Suppression of the development of uterine adenomyosis by danazol treatment in mice. Life Sci 51: 1119-1125, 1992.

20. Giudice LC and Kao LC: Endometriosis. Lancet 364: 1789-1799, 2004.

21. Gruemmer R: Animal models in endometriosis research. Hum Reprod Update 12: 641-649, 2006.

22. Mori T, Singtripop T and Kawashima S: Animal model of uterine adenomyosis: is prolactin a potent inducer of adenomyosis in mice? Am J Obstet Gynecol 165: 232-234, 1991.

23. Mori T, Nagasawa $\mathrm{H}$ and Takahashi S: The induction of adenomyosis in mice by intrauterine pituitary isografts. Life Sci 29: 1277-1282, 1981.

24. Vercellini P, Somigliana E, Vagano P, Abbiati A, Barbara G and Crosignani PG: Endometriosis - current therapies and new pharmacological developments. Drugs 69: 649-675, 2009.

25. Fang Z, Yang S, Lydon JP, DeMayo F, Tamura M, Gurates B and Bulun SE: Intact progesterone receptors are essential to counteract the proliferative effect of estradiol in a genetically engineered mouse model of endometriosis. Fertil Steril 82: 673-678, 2004

26. Bulun SE, Cheng YH, Yin P, Imir G, Utsunomiya H, Attar E, Innes J and Julie KJ: Progesterone resistance in endometriosis: link to failure to metabolize estradiol. Mol Cell Endocrinol 248: 94-103, 2006

27. Fedele L, Bianchi S and Frontino G: Hormonal treatment for adenomyosis. Best Pract Res Clin Obstet Gynaecol 22: 333-339, 2008. 